\title{
Absolute Stability of Discrete-Time Systems with Delay
}

\author{
Rigoberto Medina \\ Departamento de Ciencias Exactas, Universidad de Los Lagos, Casilla 933, Osorno, Chile
}

Correspondence should be addressed to Rigoberto Medina,rmedina@ulagos.cl

Received 18 October 2007; Accepted 22 November 2007

Recommended by Bing Gen Zhang

We investigate the stability of nonlinear nonautonomous discrete-time systems with delaying arguments, whose linear part has slowly varying coefficients, and the nonlinear part has linear majorants. Based on the "freezing" technique to discrete-time systems, we derive explicit conditions for the absolute stability of the zero solution of such systems.

Copyright ( 12008 Rigoberto Medina. This is an open access article distributed under the Creative Commons Attribution License, which permits unrestricted use, distribution, and reproduction in any medium, provided the original work is properly cited.

\section{Introduction}

Over the past few decades, discrete-time systems with delay have drawn much attention from the researchers. This is due to their important role in many practical systems. The stability of time-delay systems is a fundamental problem because of its importance in the analysis of such systems. The basic method for stability analysis is the direct Lyapunov method, for example, see [1-3], and by this method, strong results have been obtained. But finding Lyapunov functions for nonautonomous delay difference systems is usually a difficult task. In contrast, many methods different from Lyapunov functions have been successfully applied to establish stability results for difference equations with delay, for example, see [3-12].

This paper deals with the absolute stability of nonlinear nonautonomous discrete-time systems with delay, whose linear part has slowly varying coefficients, and the nonlinear part satisfies a Lipschitz condition.

The aim of this paper is to generalize the approach developed in [7] for linear nonautonomous delay difference systems to the nonlinear case with delaying arguments. Our approach is based on the "freezing" technique for discrete-time systems. This method has been used to investigate properties as well as to the construction of solutions for systems of linear 
differential equations. So, it is commonly used in analysing the stability of slowly varying initial-value problems as well as solving them, for example, see [13,14]. However, its use to difference equations is rather new [7]. The stability conditions will be formulated assuming that we know the Cauchy solution (fundamental solution) of the unperturbed system.

The paper is organized as follows. After some preliminaries in Section 2, the sufficient conditions for the absolute stability are presented in Section 3. In Section 4, we reduce a delay difference system to a delay-free linear system of higher dimension, thus obtaining explicit stability conditions for the solutions.

\section{Preliminaries}

Let $\mathbb{N}$ denote the set of nonnegative integers. Given a positive integer $n$, denote by $C^{n}$ and $M_{n}$ the $n$-dimensional space of complex column vectors and the set of $n \times n$ matrices with complex entries, respectively. If $\|\cdot\|$ is any norm on $C^{n}$, the associated induced norm of a matrix $A \in M_{n}$ is defined by

$$
\|A\|=\sup _{x \in C^{n}} \frac{\|A x\|}{\|x\|} .
$$

Consider the nonlinear discrete-time system with multiple delays of the form

$$
x(k+1)=\sum_{l=0}^{m} A_{l}(k) x(k-l)+F(k, x(k), x(k-1), \ldots, x(k-m)),
$$

where $m \geq 1$ is an integer $x(k) \in C^{n}$ and $A_{j}(k) \in M_{n}(j=0,1, \ldots, m)$.

We will consider (2.2) subject to the initial conditions

$$
x(k)=\varphi(k), \quad \text { for }-m \leq k \leq 0,
$$

where $\varphi$ is a given vector-valued function, that is, $\varphi(k) \in C^{n}$.

Throughout the paper, we will assume that the variable matrices $A_{j}(\cdot)(j=0,1, \ldots, m)$ have the properties

$$
\begin{gathered}
\left\|A_{j}(k)-A_{j}(s)\right\| \leq q_{j}|k-s|, \quad\left(q_{j}=\text { const. } \geq 0 ; k, s \in \mathbb{N}\right), \\
\sup _{s \in \mathbb{N}} \sum_{j=0}^{m}\left\|A_{j}(s)\right\|<\infty .
\end{gathered}
$$

In addition, $F: \mathbb{N} \times C^{n(m+1)} \rightarrow C^{n}$ is a given function satisfying the growth condition

$$
\left\|F\left(k, z_{0}, z_{1}, \ldots, z_{m}\right)\right\| \leq \sum_{j=0}^{m} r_{j}\left\|z_{j}\right\|,
$$

where $\gamma_{j}=$ const. $\geq 0 ; j \in \mathbb{N} ; z_{j} \in C^{n}, j=0,1, \ldots, m$.

Definition 2.1. The zero solution of (2.2) is absolutely stable in the class of nonlinearities (2.6) if there is a positive constant $M_{0}$, independent of $F$ (but dependent on $q_{0}, q_{1}, \ldots, q_{m}$ ), such that

$$
\|x(k)\| \leq M_{0} \max _{-m \leq s \leq 0}\|\varphi(s)\|, \quad s \in \mathbb{N}
$$

for any solution $x(k)$ of (2.2) with the initial conditions (2.3). 
It is clear that every solution $\{x(k)\}$ of the initial-valued problem (2.2)-(2.3) exists, is unique and can be constructed recursively from (2.2).

Put

$$
L(s) x(k)=\sum_{l=0}^{m} A_{l}(s) x(k-l) ; \quad k, s \in \mathbb{N} .
$$

The stability conditions for (2.2) will be formulated in terms of the Cauchy function $G$ (the fundamental solution) of

$$
x(k+1)-L(s) x(k)=0
$$

defined as follows. For a fixed $s \in \mathbb{N}$, let $\{G(k, s)\}_{k=s-m}^{\infty}$ be the solution of (2.9) with initial conditions

$$
G(k, s)= \begin{cases}0 & \text { for } s-m \leq k \leq s-1, \\ 1 & \text { for } k=s .\end{cases}
$$

Since the coefficients of (2.9) are constants for fixed $s \in \mathbb{N}$, then the Cauchy function of (2.9) has the form

$$
G(k, s)=v(k-s), \quad \text { for } k \geq s-m,
$$

where $v$ is the solution of (2.9) with the initial conditions

$$
v(k)= \begin{cases}0, & \text { for }-m \leq k \leq-1, \\ 1 & \text { for } k=0\end{cases}
$$

In order to state and prove our main results, we need some suitable lemmas and theorems.

Lemma 2.2 (see [7]). The solution $\{x(k)\}_{k=k_{0}-m}^{\infty}$ of

$$
x(k+1)=\sum_{l=0}^{m} A_{l}(k) x(k-l)+f(k),
$$

where $f: \mathbb{N} \rightarrow C^{n}$ is a given function, subject to the initial conditions

$$
x(k)=\varphi(k), \quad \text { for } k_{0}-m \leq k \leq k_{0},
$$

has the form

$$
x(k)=y(k)+\sum_{j=k_{0}}^{k-1} G(k, j+1) f(j), \quad \text { for } k \geq k_{0}
$$

where $G$ is the Cauchy function of (2.9) and $\{y(k)\}_{k=k_{0}-m}^{\infty}$ is the solution of the homogeneous equation

$$
x(k+1)=\sum_{l=0}^{m} A_{l}(k) x(k-l)
$$

with the same initial conditions:

$$
y(k)=\varphi(k), \quad \text { for } k_{0}-m \leq k \leq k_{0} .
$$


Lemma 2.3 (see [7]). The solution $\{y(k)\}_{k=k_{0}-m}^{\infty}$ of (2.16) with initial conditions (2.14) has the form

$$
y(k)=G\left(k, k_{0}\right) \varphi\left(k_{0}\right)+\sum_{i=1}^{m} \sum_{j=k_{0}}^{k_{0}+i-1} A_{i}(j) G(k, j+1) \varphi(j-i), \quad k \geq k_{0} .
$$

In [7], was established the following stability result in terms of the Cauchy solution G of (2.9).

Theorem 2.4 (see [7]). Let the inequality

$$
\|G(k, s)\| \leq N \eta^{k} \quad(k, s \in \mathbb{N})
$$

holds with constant $\eta \in(0,1)$, and $N$ independent of s. If in addition, conditions (2.4), (2.5), and $N \tilde{q}<(1-\eta)^{2}$ are fulfilled, then (2.16) is stable.

Our purpose is to generalize this result to the nonlinear problem (2.2)-(2.3).

Lemma 2.5 (see [9]). Let $\{g(k)\}_{k=k_{0}}^{\infty}$ be a sequence of positive numbers such that

$$
\sum_{j=k_{0}}^{k-1} \frac{g(k)}{g(j+1)} \leq \Gamma \quad \text { for } k \geq k_{0}
$$

where $\Gamma>0$ is a constant. Then there exist constants $\alpha>0$ and $\lambda \in(0,1)$ such that

$$
g(k) \leq \alpha \lambda^{k-k_{0}} \quad \text { for } k \geq k_{0}
$$

\section{Main results}

Now, we establish the main results of the paper, which will be valid for a family $\left\{A_{j}(k)\right\}_{k=0}^{\infty}$ $(j=0,1, \ldots, m)$ of slowly varying matrices. Let $\tilde{q}=\sum_{i=0}^{m} q_{i}$ and $\tilde{\gamma}=\sum_{i=0}^{m} \gamma_{i}$. With the notation

$$
\psi(k)=\sup _{s \in \mathbb{N}}\|G(k, s)\|,
$$

assume that

$$
\tilde{\psi}_{0}=\sum_{k=0}^{\infty} \psi(k)<\infty, \quad \tilde{\psi}_{1}=\sum_{k=1}^{\infty} k \psi(k)<\infty
$$

Consider the equation

$$
x(k+1)-L(k) x(k)=f(k),
$$

where $f: \mathbb{N} \rightarrow C^{n}$ is a bounded function such that

$$
\|f\|_{\infty}=\sup _{k \in \mathbb{N}}\|f(k)\|<\infty .
$$


Theorem 3.1. Under conditions (2.4) and (2.5), let the inequality

$$
\tilde{q} \tilde{\varphi}_{1}<1
$$

holds. Then for any solution $x(k)$ of problem (2.13)-(2.3), the estimate

$$
\|x(k)\| \leq C_{0}\|\varphi\|+\tilde{\varphi}_{0}\left(1-\tilde{q} \widetilde{\varphi}_{1}\right)^{-1}\|f\|_{\infty}
$$

is valid, where $C_{0}=$ const., and $\|\varphi\|=\max _{-m \leq k \leq 0}\|\varphi(k)\|$.

Proof. Fix $s \geq 0$ and rewrite (3.3) in the form

$$
x(k+1)-L(s) x(k)=(L(k)-L(s)) x(k)+f(k) .
$$

Making

$$
H(k, s)=(L(k)-L(s)) x(k)+f(k)
$$

we get

$$
x(k+1)-L(s) x(k)=H(k, s) .
$$

A solution of the latter equation, subject to the initial conditions (2.3), can be represented as

$$
x(k)=y_{s}(k)+\sum_{j=0}^{k-1} G(k-j-1) H(j, s), \quad k \geq 0
$$

where $y_{s}(k)$ is the solution of the homogeneous equation (2.9) with initial conditions (2.3). Since $y_{s}(k)$ is a solution of (2.9), we can write

$$
\begin{aligned}
y_{s}(k) & =G(k, 0) \varphi(0)+\sum_{i=0}^{m} A_{i}(s) \sum_{j=0}^{i-1} G(k-j-1) \varphi(j-i) \\
& =G(k, 0) \varphi(0)+\sum_{i=0}^{m} A_{i}(s) \sum_{\tau=-i}^{-1} G(k-\tau-i-1) \varphi(\tau) .
\end{aligned}
$$

This relation and (2.5) yield

$$
\left\|y_{s}(k)\right\| \leq c_{1}<\infty \quad\left(c_{1}=\text { const.; } k, s \geq 0\right),
$$

since the Cauchy function is bounded by (3.2). Moreover,

$$
c_{1} \leq c_{2} \max _{-m \leq k \leq 0}\|\varphi(k)\| \quad\left(c_{2}=\text { const. }\right) .
$$


From (3.10), it follows that

$$
\begin{aligned}
\|x(k)\| & \leq\left\|y_{s}(k)\right\|+\sum_{j=0}^{k-1}\|G(k-j-1)\|\|H(j, s)\| \\
& \leq c_{1}+\sum_{j=0}^{k-1}\|G(k-j-1)\|\|H(j, s)\| .
\end{aligned}
$$

According to (2.4), we have

$$
\begin{aligned}
\|H(j, s)\| & \leq \sum_{k=0}^{m}\left\|\left(A_{k}(j)-A_{k}(s) x(j-k)\right)+f(j)\right\| \\
& \leq \sum_{k=0}^{m}\left\|A_{k}(j)-A_{k}(s)\right\|\|x(j-k)\|+\|f\|_{\infty} \\
& \leq \sum_{k=0}^{m} q_{k}|j-s|\|x(j-k)\|+\|f\|_{\infty} .
\end{aligned}
$$

Take $k=s$. Then, by the estimate

$$
\sum_{j=0}^{k-1} \psi(k-j-1)\|f(j)\| \leq c(f) \equiv \tilde{\psi}_{0}\|f\|_{\infty \prime}
$$

it follows that

$$
\begin{aligned}
\|x(k)\| & \leq c_{1}+c(f)+\sum_{j=0}^{k-1} \psi(k-j-1)\left(\sum_{i=0}^{m} q_{i}|k-j|\|x(j-i)\|\right) \\
& \leq c_{1}+c(f)+\sum_{i=0}^{m} q_{i} \sum_{z=-i}^{k-i-1} \psi(k-z-i)|k-z-i|\|x(z)\| .
\end{aligned}
$$

Hence,

$$
\|x(k)\| \leq \sum_{i=0}^{m} q_{i} \sum_{z=0}^{k-i-1} \psi(k-z-i)|k-z-i|\|x(z)\|+c_{3}(f),
$$

where

$$
c_{3}(f)=c_{1}+c(f)+\sup _{k \in \mathbb{N}} \sum_{i=0}^{m} q_{i} \sum_{z=-i}^{0} \psi(k-z-i)|k-z-i|\|\varphi(z)\| .
$$

Making

$$
M\left(k_{0}\right)=\max _{0 \leq k \leq k_{0}}\|x(k)\|
$$

we obtain

$$
\begin{aligned}
M\left(k_{0}\right) & \leq c_{3}(f)+M\left(k_{0}\right) \sum_{i=0}^{m} q_{i} \sum_{z=0}^{k-i-1} \psi(k-z-i)|k-z-i| \\
& \leq c_{3}(f)+M\left(k_{0}\right) \tilde{q} \widetilde{\psi}_{1} .
\end{aligned}
$$


Condition (3.5) implies the inequality

$$
M\left(k_{0}\right) \leq c_{3}(f)\left(1-\tilde{q} \widetilde{\psi}_{1}\right)^{-1} .
$$

Since $k_{0}$ is arbitrary, we obtain the estimate

$$
\sup _{k \geq 0}\|x(k)\| \leq c_{3}(f)\left(1-\tilde{q} \widetilde{\psi}_{1}\right)^{-1}
$$

Further,

$$
c_{3}(f) \leq c_{4} \max _{-m \leq k \leq 0}\|\varphi(k)\|+\widetilde{\psi}_{0}\|f\|_{\infty} ; \quad\left(c_{4}=\text { const. }\right) .
$$

This yields the required result.

Corollary 3.2. Under conditions (2.4) and (2.5), let the inequality

$$
\|G(k, s)\| \leq N \eta^{k} \quad(k, s \in \mathbb{N})
$$

hold, with constants $\eta \in(0,1)$ and $N$ independent of s. If, in addition,

$$
N \tilde{q}<(1-\eta)^{2} .
$$

Then, any solution $x(k)$ of (2.13)-(2.3) satisfies the estimate

$$
\|x(k)\| \leq b_{0}\|\varphi\|+\frac{N}{1-\eta}\left(1-\frac{N}{(1-\eta)^{2}} \tilde{q}\right)^{-1}\|f\|_{\infty}
$$

where $\|\varphi\|=\max _{-m \leq k \leq 0}\|\varphi(k)\|$, and $b_{0}=$ const.

Proof. Under condition (3.25), we obtain

$$
\begin{aligned}
& \tilde{\Psi}_{0} \leq N \sum_{k=0}^{\infty} \eta^{k}=\frac{N}{1-\eta^{\prime}} \\
& \tilde{\Psi}_{1} \leq N \sum_{k=0}^{\infty} k \eta^{k}=\frac{N}{(1-\eta)^{2}} .
\end{aligned}
$$

Now, Corollary 3.2 yields the following result.

Theorem 3.3. Let the conditions (2.4), (2.5), (2.6), (3.25), and, in addition,

$$
N\left(\frac{\tilde{\gamma}}{1-\eta}+\frac{\tilde{q}}{(1-\eta)^{2}}\right)<1
$$

hold. Then, the zero solution of (2.2)-(2.3) is absolutely stable in the class of nonlinearities in (2.6). 
Proof. Condition (3.29) implies the inequality (3.26), and in addition

$$
\frac{\tilde{\gamma} N}{1-\eta}\left(1-\frac{N \tilde{q}}{(1-\eta)^{2}}\right)^{-1}<1
$$

By (2.6), we obtain

$$
\|F(k, x(k), x(k-1), \ldots, x(k-m))\| \leq \tilde{\gamma}\|x\|_{[-m, \infty)} \leq \tilde{\gamma}\left(\|x\|_{[0, \infty)}+\|\varphi\|\right),
$$

where $x(k)$ is a solution of $(2.2)$ and $[-m, \infty): \equiv\{-m,-m+1, \ldots, 0,1, \ldots\}$.

Let

$$
f(k)=F(k, x(k), x(k-1), \ldots, x(k-m)),
$$

then (2.2) takes the form (3.3). Thus, Corollary 3.2 implies

$$
\|x(k)\| \leq b_{0}\|\varphi\|+\frac{N}{1-\eta}\left(1-\frac{N}{(1-\eta)^{2}} \tilde{q}\right)^{-1}\left(\|x\|_{[0, \infty)}+\|\varphi\|\right) .
$$

Thus, condition (3.29) implies

$$
\|x(k)\| \leq M_{0}\|\varphi\|\left[1-\frac{\tilde{\gamma} N}{1-\eta}\left(1-\frac{N \tilde{q}}{(1-\eta)^{2}}\right)^{-1}\right]^{-1},
$$

where

$$
M_{0}=b_{0}+\frac{\tilde{\gamma} N}{1-\eta}\left(1-\frac{N \tilde{q}}{(1-\eta)^{2}}\right)^{-1} .
$$

This fact proves the required result.

Remark 3.4. Theorem 3.3 is exact in the sense that if (2.2) is a homogeneous linear stable equation with constant matrices $A_{j}(k) \equiv A_{j}$, then $\tilde{q}=\tilde{\gamma}=0$, and condition (3.29) is always fulfilled.

It is somewhat inconvenient that to apply either condition (3.26) or (3.29), one has to assume explicit knowledge of the constants $N$ and $\eta$. In the next theorem, we will derive sufficient conditions for the exponential growth of the Cauchy function associated to (2.9). Thus, our conditions may provide a useful tool for applications.

Theorem 3.5 (see [7]). Assume that the Cauchy function $G\left(k, k_{0}\right)$ of $(2.9)$ satisfies

$$
\sum_{j=k_{0}}^{k-1} \frac{\left\|G\left(k, k_{0}\right)\right\|}{\left\|G\left(j+1, k_{0}\right)\right\|} \leq \Gamma \quad \text { for } k \geq k_{0}
$$

where $\Gamma>0$ is a constant. Then there exist constants $\beta>0$ and $0<\lambda<1$ such that

$$
\left\|G\left(k, k_{0}\right)\right\| \leq \beta \lambda^{k-k_{0}} \quad \text { for } k \geq k_{0} .
$$


Now, we will consider the homogeneous equation (2.16), thus establishing the following consequence of Theorem 3.3.

Corollary 3.6. Let conditions (2.4), (2.5), (3.25), and, in addition,

$$
\frac{N \tilde{q}}{(1-\eta)^{2}}<1
$$

hold. Then the zero solution of (2.16)-(2.3) is absolutely stable.

Example 3.7. Consider the following delay difference system in the Euclidean space $R^{2}$ :

$$
x(k+1)=A_{0}(k) x(k)+A_{1}(k) x(k-1)+F(k, x(k), x(k-1)), \quad k \in \mathbb{N},
$$

where

$$
A_{0}(k)=\left(\begin{array}{cc}
a_{0}(k) & b_{0}(k) \\
c_{0}(k) & 0
\end{array}\right), \quad A_{1}(k)=\left(\begin{array}{cc}
a_{1}(k) & b_{1}(k) \\
c_{1}(k) & d_{1}(k)
\end{array}\right),
$$

and $x(k) \in R^{2}$. And $a_{i}(k), b_{i}(k), c_{i}(k), d_{i}(k), i=0,1$, are positive bounded sequences with the following properties: $\left\|A_{0}(k+1)-A_{0}(k)\right\| \leq q_{0}$ and $\left\|A_{1}(k+1)-A_{1}(k)\right\| \leq q_{1}$ and $q_{i} ; i=0,1$, are nonnegative constants for $k \in \mathbb{N}$. This yields that $\left\|A_{0}(k)-A_{0}(s)\right\| \leq q_{0}$ and $\left\|A_{1}(k)-A_{1}(s)\right\| \leq q_{1}$, respectively, for $k, s \in \mathbb{N}$. Thus $\tilde{q}=q_{0}+q_{1}$.

condition

In addition, the function $F: \mathbb{N} \times R^{2} \times R^{2} \rightarrow R^{2}$ supplies the solvability and satisfies the

$$
\|F(k, u, v)\| \leq \gamma_{0}\|u\|+\gamma_{1}\|v\| ; \quad u, v \in R^{2}, k \in \mathbb{N} .
$$

Hence, $\tilde{\gamma}=\gamma_{0}+\gamma_{1}$.

Further, assume that the Cauchy solution $G(k, s)$ of equation

$$
x(k+1)=\left(\begin{array}{cc}
a_{0}(s) & b_{0}(s) \\
c_{0}(s) & 0
\end{array}\right) x(k)+\left(\begin{array}{ll}
a_{1}(s) & b_{1}(s) \\
c_{1}(s) & d_{1}(s)
\end{array}\right) x(k-1)
$$

for a fixed $s \in \mathbb{N}$ tends to zero exponentially as $k \rightarrow \infty$, that is, there exist constants $N>0$ and $\eta \in(0,1)$ such that $\|G(k, s)\| \leq N \eta^{k} ; k \in \mathbb{N}$.

If $N\left(\tilde{\gamma} /(1-\eta)+\tilde{q} /(1-\eta)^{2}\right)<1$, then by Theorem 3.3, it follows that the zero solution of (3.39) is absolutely stable.

For instance, if the linear system with constant coefficients associated to the nonlinear system with variable coefficients (3.39) is

$$
x(k+1)=\left(\begin{array}{ll}
-0.1 & 0.3 \\
-0.5 & 0.0
\end{array}\right) x(k)+\left(\begin{array}{cc}
0.7 & -0.4 \\
0.5 & -0.8
\end{array}\right) x(k-1), \quad k \in \mathbb{N},
$$

then it is not hard to check that the Cauchy solution of this system tends to zero exponentially as $k \rightarrow \infty$. Hence, by Theorem 3.3, it follows that the zero solution of (3.39) is absolutely stable provided that the relation (3.29) is satisfied. 


\section{Linear delay systems}

Now, we will consider an important particular case of (2.2), namely, the linear delay difference system

$$
x(k+1)=A(k) x(k)+B(k) x(k-m) ; \quad k \in \mathbb{N},
$$

where $x(k) \in C^{n}, A(k)$, and $B(k)$ are variable $n \times n$-matrices.

In [4], were established very nice solution representation formulae to the system

$$
x(k+1)=A x(k)+B x(k-m)+f(k),
$$

assuming that $A B=B A$ and $\operatorname{det} A \neq 0$. However, the stability problem was not investigated in this paper.

Kipnis and Komissarova [6] investigated the stability of the system

$$
x_{n}=A x_{n-1}+B x_{n-k}
$$

where $A, B$ are $m \times m$-matrices, $x_{n} \in R^{m}$. By means of a characteristic equation, they established many results concerning the stability of the solutions of such equation. However, the case of variable coefficients is not studied in this article.

In the next corollary, we will apply Theorem 3.3 to this particular case of (2.2), thus obtaining the following corollary.

Corollary 4.1. Under condition (3.25), one assumes that

(i) the matrices $A(k)$ and $B(k)$ satisfy $\|A(k)-A(s)\| \leq q_{0}|k-s|$ and $\|B(k)-B(s)\| \leq q_{1}|k-s|$, respectively, for $k, s \in \mathbb{N}$;

(ii) $\sup _{k \in \mathbb{N}}(\|A(k)\|+\|B(k)\|)<\infty$;

(iii)

$$
\frac{N\left(q_{0}+q_{1}\right)}{(1-\eta)^{2}}<1
$$

Then, the zero solution of (4.1)-(2.3) is absolutely stable.

Remark 4.2. I want to point out that this approach is just of interest for systems with "slowly changing" matrices.

The purpose of this section is to apply a new method to investigate the stability of system (4.1), which combined with the "freezing technique," will allow us to derive explicit estimations to their solutions, namely, introducing new variables; one can reduce system (4.1) to a delay-free linear difference system of higher dimension. In fact, put

$$
u_{1}=x(k), u_{2}=x(k-1), \ldots, u_{m+1}=x(k-m) \text {. }
$$

Then (4.1) takes the form

$$
w(k+1)=T(k) w(k), \quad k \in \mathbb{N},
$$


where

$$
\begin{aligned}
w(k) & =\operatorname{col}\left(u_{1}(k), u_{2}(k), \ldots, u_{m+1}(k)\right), \\
T(k) & =\left[\begin{array}{ccccc}
A(k) & 0 & \cdots & 0 & B(k) \\
I & 0 & \cdots & 0 & 0 \\
0 & I & \cdots & 0 & 0 \\
\vdots & \vdots & \cdots & \vdots & \vdots \\
0 & 0 & \cdots & I & 0
\end{array}\right]_{(m+1) \times(m+1)}
\end{aligned}
$$

where $I$ is the unit matrix in $C^{n}$.

Let $C^{n(m+1)}$ be the product of $(m+1)$ copies of $C^{n}$. Then we can consider (4.6) defined in the space $C^{n(m+1)}$. In $C^{n(m+1)}$, define the norm

$$
\|v\|_{C^{n(m+1)}}=\left[\sum_{k=1}^{m+1}\left\|v_{k}\right\|_{C^{n}}^{2}\right]^{1 / 2} \quad \text { for } v=\operatorname{col}\left(v_{1}, v_{2}, \ldots, v_{m+1}\right) \in C^{n(m+1)}
$$

For an $n \times n$-matrix $A$, denote

$$
g(A)=\left[N^{2}(A)-\sum_{j=1}^{n}\left|\lambda_{j}(A)\right|^{2}\right]^{1 / 2}
$$

where $N(A)$ is the Frobenius (Hilbert-Schmidt) norm of a matrix $A, N^{2}(A)=$ Trace $\left(A A^{*}\right)$, and $\lambda_{1}(A), \lambda_{2}(A), \ldots, \lambda_{n}(A)$ are the eigenvalues of $A$, including their multiplicities. Here $A^{*}$ is the adjoint matrix. If $A$ is normal, that is, $A^{*} A=A A^{*}$, then $g(A)=0$. If $A=\left(a_{i j}\right)$ is a triangular matrix such that $a_{i j}=0$ for $1 \leq j \leq i \leq n$, then

$$
g^{2}(A)=\sum_{1 \leq j \leq i \leq n}\left|a_{i j}\right|^{2}
$$

Due to $[15$, Theorem 2.1], for any $n \times n$-matrix $A$, the inequality

$$
\left\|A^{m}\right\| \leq \sum_{k=0}^{m_{1}} \frac{m ! \rho^{m-k}(A) g^{k}(A)}{(m-k) !(k !)^{3 / 2}}, \quad m_{1}=\min \{n-1, m\}
$$

holds for every nonnegative integer $m$, where $\rho(A)$ is the spectral radius of $A$.

Theorem 4.3 (see [7]). Assume that

(i) $\|T(k)-T(j)\|_{C^{n(m+1)}} \leq q|k-j| ; k, j \in \mathbb{N}$ and $q=$ const. $>0$;

(ii) $\beta_{0}=\sup _{k, l=0,1, \ldots}\left\|T^{k}(l)\right\|_{C^{n(m+1)}}<\infty, \mu_{0}=\sum_{k=0}^{\infty} k \sup _{l=1,2, . .}\left\|T^{k}(l)\right\|_{C^{n(m+1)}}<q^{-1}$.

Then, any solution $\{x(k)\}$ of (4.1) is bounded and satisfies the inequality

$$
\sup _{k=1,2, \ldots}\|x(k)\|_{C^{n}} \leq \beta_{0}\|w(0)\|_{C^{n(m+1)}}\left(1-\mu_{0} q\right)^{-1},
$$

where $w(0)=(\varphi(0), \varphi(-1), \ldots, \varphi(-m))$, with $\varphi$ defined in (2.14). 
Since the calculation of quantities $\beta_{0}$ and $\mu_{0}$ is not an easy task, by (4.11), some estimations to these formulae, namely, in terms of the eigenvalues of auxiliary matrices will be driven. In doing so, one assumes that

$$
\rho_{0}=\sup _{l=1,2, \ldots} \rho(T(l))<1, \quad v_{0}=\sup _{l=0,1, \ldots} g(T(l))<\infty,
$$

and denote

$$
M=\sum_{j=0}^{n(m+1)-1} \frac{\left(\psi_{j}+j\right)^{j} \rho_{0}^{\psi_{j}} v_{0}^{j}}{(j !)^{3 / 2}}
$$

where $\psi_{j}=\max \left\{0, j\left(1 /\left(\ln \left(1 / \rho_{0}\right)\right)-1\right)\right\}$ and $\theta_{0}=\sum_{k=0}^{n(m+1)-1}\left((k+1) v_{0}^{k}\right) /\left(\sqrt{k !}\left(1-\rho_{0}\right)^{k+2}\right)$.

Corollary 4.4. Under condition (i) of Theorem 4.3, let (4.13) and $q \theta_{0}<1$ hold. Then, any solution $x(k)$ of (4.1) is bounded. Moreover,

$$
\sup _{k=1,2, \ldots}\|x(k)\|_{C^{n(m+1)}} \leq M\|w(0)\|_{C^{n(m+1)}}\left(1-q \theta_{0}\right)^{-1}
$$

where $w(0)=(\varphi(0), \varphi(-1), \ldots, \varphi(-m))$.

Proof. By (4.11), we obtain

$$
\beta_{0} \leq \sup _{k=1,2, \ldots} \sum_{j=0}^{n(m+1)-1} \frac{k ! \rho_{0}^{k-j} v_{0}^{j}}{(k-j) !(j !)^{3 / 2}}
$$

The relation

$$
\frac{k !}{(k-j) !} \leq k^{j}, \quad k \geq j
$$

implies that

$$
\sup _{k=j, j+1, \ldots} k^{j} \rho_{0}^{k-j} \leq \max _{x \geq 0}\left\{(x+j)^{j} \rho_{0}^{x}\right\}
$$

Simple calculations show that

$$
\max _{x \geq 0}\left\{(x+j)^{j} \rho_{0}^{x}\right\}=\left(\psi_{j}+j\right)^{j} \rho_{0}^{\psi_{j}} \quad \text { for } k=1,2, \ldots
$$

Thus,

$$
\frac{k ! \rho_{0}^{k-j}}{(k-j) !} \leq\left(\psi_{j}+j\right)^{j} \rho_{0}^{\psi_{j}} \quad \text { for } k=1,2, \ldots
$$

Hence, $\beta_{0} \leq M$. 
On the other hand,

$$
\mu_{0} \leq \sum_{k=0}^{\infty} \sum_{j=0}^{n(m+1)-1} \frac{k k ! \rho_{0}^{k-j} v_{0}^{j}}{(k-j) !(j !)^{3 / 2}}
$$

But

$$
\begin{aligned}
\sum_{k=1}^{\infty} \frac{k k ! z^{k-j}}{(k-j) !} & \leq \sum_{k=1}^{\infty} \frac{(k+1) ! z^{k-j}}{(k-j) !} \\
& =\frac{d^{j+1}}{d z^{j+1}} \sum_{k=1}^{\infty} z^{k+1}=\frac{d^{j+1}}{d z^{j+1}} z(1-z)^{-1} \\
& =(j+1) !(1-z)^{-j-2} \text { for } 0<z<1 ; j=0,1, \ldots, n(m+1) .
\end{aligned}
$$

Thus, it follows that

$$
\mu_{0} \leq \sum_{k=0}^{\infty} \sum_{j=0}^{n(m+1)-1} \frac{k k ! \rho_{0}^{k-j} v_{0}^{j}}{(k-j) !(j !)^{3 / 2}} \leq \sum_{j=0}^{n(m+1)-1} \frac{(j+1) v_{0}^{j}}{\sqrt{j !}\left(1-\rho_{0}\right)^{j+2}}=\theta_{0} .
$$

Remark 4.5. This approach is usually not applicable to the time-varying delay case, because the transformed systems usually have time-varying matrix coefficients, which are difficult to analyze using available tools. Hence, our results will provide new tools to analyze these kind of systems.

\section{Acknowledgments}

The author thanks the referees of this paper for their careful reading and insightful critiques. This research was supported by Fondecyt Chile under Grant no. 1.070.980.

\section{References}

[1] R. P. Agarwal, Difference Equations and Inequalities. Theory, Methods, and Applications, vol. 155 of Monographs and Textbooks in Pure and Applied Mathematics, Marcel Dekker, New York, NY, USA, 1992.

[2] V. Lakshmikantham and D. Trigiante, Theory of Difference Equations, vol. 181 of Mathematics in Science and Engineering, Academic Press, Boston, Mass, USA, 1988.

[3] M. Vidyasagar, Nonlinear Syatems Analysis, Prentice-Hall, Englewood-Cliffs, NJ, USA, 1978.

[4] J. Diblík and D. Ya. Khusainov, "Representation of solutions of discrete delayed system $x(k+1)=$ $A x(k)+B x(k-m)+f(k)$ with commutative matrices," Journal of Mathematical Analysis and Applications, vol. 318, no. 1, pp. 63-76, 2006.

[5] S. Elayi and S. Zhang, "Stability and periodicity of difference equations with finite delay," Funkcialaj Ekvacioj, vol. 37, no. 3, pp. 401-413, 1994.

[6] M. Kipnis and D. Komissarova, "Stability of delay difference system," Advances in Difference Equations, vol. 2006, Article ID 31409, 9 pages, 2006.

[7] R. Medina, "Stability analysis of nonautonomous difference systems with delaying arguments," Journal of Mathematical Analysis and Applications, vol. 335, no. 1, pp. 615-625, 2007.

[8] M. Pituk, "Convergence and uniform stability in a nonlinear delay difference system," Mathematical and Computer Modelling, vol. 22, no. 2, pp. 51-57, 1995. 
[9] M. Pituk, "Global asymptotic stability in a perturbed higher-order linear difference equation," Computers \& Mathematics with Applications, vol. 45, no. 6-9, pp. 1195-1202, 2003.

[10] J. Schinas, "Stability and conditional stability of time-dependent difference equations in Banach spaces," Journal of the Institute of Mathematics and Its Applications, vol. 14, no. 3, pp. 335-346, 1974.

[11] S. Zhang, "Estimate of total stability of delay difference systems," Computers \& Mathematics with Applications, vol. 37, no. 9, pp. 31-38, 1999.

[12] S. A. Kuruklis, "The asymptotic stability of $x_{n+1}-a x_{n}+b x_{n-k}=0$," Journal of Mathematical Analysis and Applications, vol. 188, no. 3, pp. 719-731, 1994.

[13] S. M. Shahruz and A. L. Schwarz, "An approximate solution for linear boundary-value problems with slowly varying coefficients," Applied Mathematics and Computation, vol. 60, no. 2-3, pp. 285-298, 1994.

[14] R. Qu and R. P. Agarwal, "Improved error bounds for freezing solutions of linear boundary value problems," Applied Mathematics and Computation, vol. 94, no. 2-3, pp. 97-112, 1998.

[15] M. I. Gil and R. Medina, "The freezing method for linear difference equations," Journal of Difference Equations and Applications, vol. 8, no. 5, pp. 485-494, 2002. 